\title{
TEMPERATURAS DE ACONDICIONAMIENTO, POSCOSECHA SOBRE EL DESARROLLO DE COLOR DE LA EPIDERMIS Y CALIDAD INTERNA DE FRUTOS DE PIÑA
}

\author{
Luis Ulloa-Gómez ${ }^{1}$, Marco Vinicio Sáenz-Murillo2/*, \\ Johanny Castro-Chinchilla ${ }^{3}$, Maricruz Ramirez-Sánchez ${ }^{4}$ \\ Palabras clave: Temperaturas de acondicionamiento; índice de color; \\ respiración; no-climatérico; pérdida de peso. \\ Keywords: Acclimatization temperatures; color index; respiration; non-climacteric; weight loss.
}

Recibido: 09/03/2020

Aceptado: $23 / 06 / 2020$

\section{RESUMEN}

Introducción. Los frutos de piña ofrecidos en el mercado final muestran pobre desarrollo de color, debido en parte a que la clorofila se degrada muy lentamente durante el almacenamiento en frío. Objetivo. Evaluar el efecto de la temperatura de acondicionamiento previo a simulación de transporte y posterior almacenamiento, sobre el desarrollo de color amarillo en la epidermis de frutos de piña cv. Dorada Extra Dulce. Materiales y métodos. Frutos de piña fueron expuestos a temperaturas de $7,5^{\circ} \mathrm{C}$; $10,0^{\circ} \mathrm{C} ; 12,5^{\circ} \mathrm{C} ; 15,0^{\circ} \mathrm{C} ; 17,5^{\circ} \mathrm{C}$ y $20,0^{\circ} \mathrm{C}$ por 48 horas y luego almacenados $\mathrm{C}$ durante 21 días a $7,5^{\circ}$. Se determinó su efecto sobre índice de color $\left((1000 * a) /\left(L^{*} b\right)\right)$ de la epidermis, tasa respiratoria, producción de etileno, pérdida de peso, desarrollo de mohos en el pedúnculo y calidad interna de las frutas (firmeza, sólidos solubles, acidez

\begin{abstract}
Conditioning temperatures, at postharvest stage, on color development of the peel and internal quality of pineapple fruit. Introduction. Pineapple fruits offered at final markets show poor external color development, due in part because chlorophyll degrades very slowly during cold storage. Objective. To evaluate the effect of the conditioning temperature prior to transport simulation and subsequent storage on the development of yellow color in the epidermis of pineapple fruits cv. Golden Extra Sweet. Materials and methods. Pineapple fruits were exposed to $7.5^{\circ} \mathrm{C}, 10.0^{\circ} \mathrm{C}, 12.5^{\circ} \mathrm{C}$, $15.0^{\circ} \mathrm{C}, 17.5^{\circ} \mathrm{C}$ and $20.0^{\circ} \mathrm{C}$ for 48 hours and then stored for 21 days at $7.5^{\circ} \mathrm{C}$. Its effect on color index $\left(\left(1000^{*} \mathrm{a}\right) /\left(\mathrm{L}^{*} \mathrm{~b}\right)\right)$, respiratory rate, ethylene production, weight loss, development of molds on the peduncle and internal fruit quality (firmness,
\end{abstract}

\footnotetext{
* Autor para correspondencia. Correo electrónico: marco.saenz@ucr.ac.cr

1 Universidad de Costa Rica, Centro de Investigaciones Agronómicas, Laboratorio de Tecnología de Poscosecha, San José, Costa Rica. (D) 0000-0002-9268-2832.

2 Universidad de Costa Rica, Centro de Investigaciones Agronómicas, Laboratorio de Tecnología de Poscosecha, San José, Costa Rica. (iD) 0000-0001-6718-0622.
}
3 Universidad de Costa Rica, Centro de Investigaciones Agronómicas, Laboratorio de Tecnología de Poscosecha, San José, Costa Rica. (iD) 0000-0003-4866-3295.
4 Universidad de Costa Rica, Centro de Investigaciones Agronómicas, Laboratorio de Tecnología de Poscosecha, San José, Costa Rica. (iD) 0000-0002-8933-7931.


titulable y translucidez). Resultados. La temperatura de acondicionamiento presentó efecto lineal en el índice de color de la epidermis, tanto al final de las 48 horas de acondicionamiento como al final de los 21 días de almacenamiento a $7,5^{\circ} \mathrm{C}$. La tasa respiratoria mostró un incremento exponencial con el aumento de las temperaturas de acondicionamiento y no se evidenció efecto de las mismas sobre la producción de etileno. La pérdida de peso y el desarrollo de mohos en el pedúnculo se incrementaron con el aumento en las temperaturas de acondicionamiento durante 48 horas. La calidad interna no se afectó por las diferentes temperaturas evaluadas previas al almacenamiento en frío. Conclusiones. Mantener frutos de piña durante 48 horas a temperaturas entre $15^{\circ} \mathrm{C}$ y $20,0^{\circ} \mathrm{C}$ previo al almacenamiento a $7,5^{\circ} \mathrm{C}$, incrementaron el índice de color de la epidermis de la fruta, sin afectar la calidad interna de la misma, pero temperaturas superiores a $15^{\circ} \mathrm{C}$ indujeron aumento de incidencia y severidad de mohos.

\section{INTRODUCCIÓN}

Dentro de los diferentes parámetros que definen la calidad de los productos hortofrutícolas se encuentran la forma, tamaño y color externo. Según Brat et al. (2004) y Garita-Coto (2014), el color externo de la piña representa el principal aspecto de calidad en los mercados internacionales, el cual se requiere que se encuentre en más de un $80 \%$ de coloración amarilla en la epidermis de la fruta.

El cambio de color de verde a amarillo, en la epidermis de las piñas, involucra la degradación de clorofila y la expresión de carotenoides y antocianinas que fueron acumulados durante las últimas semanas de desarrollo del fruto (Brat et al. 2004, Ulloa et al. 2015). La degradación de la clorofila, es un proceso catabólico importante durante la maduración de los frutos, soluble solids, titratable acidity and translucency) was determined. Results. Pineapple exposed to different acclimatization temperatures before storage showed a linear increase on color index of the peel, both at the end of the 48 hours and at the end of the 21 days at $7.5^{\circ} \mathrm{C}$. Respiratory rate showed an exponential increase with increasing temperatures, and there was no evidence of their effect on ethylene production. Weight loss and mold development on the peduncle increased with increasing acclimatization temperatures for 48 hours. Internal quality was not affected by evaluated temperatures prior to cold storage. Conclusions. Keeping pineapple fruits for 48 hours at temperatures between 15.0 and $20.0^{\circ} \mathrm{C}$ prior to storage at $7.5^{\circ} \mathrm{C}$, increased peel color index without affecting its internal quality. However, exposure of the fruit at temperatures above $15^{\circ} \mathrm{C}$ increased the incidence and severity on molds.

en el que intervienen diferentes enzimas. Las clorofilasas son las primeras en actuar y forman la clorofilida $a$, que sirve de sustrato para la magnesio dequelatasa, que produce Feorforbido $a$, que es atacado por la Feoforbido $a$ Oxigenasa (PAO, paso regulatorio) y forma Catabolitos Fluorescentes de Clorofila (FCC), en el cloroplasto. Posteriormente, los FCC deben pasar a la vacuola para ser transformados en Catabolitos no Fluorescentes de Clorofila ( Du et al. 2014, Guevara y Jiménez 2006).

La degradación de clorofila, así como la síntesis o la expresión de carotenoides y antocianinas, en la epidermis de los frutos está regulado por la acción de etileno y por las temperaturas a las cuales se exponen. Por ejemplo en cítricos, la aplicación de etileno incrementa la expresión y actividad de genes involucrados en la producción de enzimas 
relacionadas a los procesos de degradación de clorofila y síntesis de carotenoides, lo que ocasiona mayor pérdida de color verde y ganancia de color anaranjado (Ma et al. 2015, Rodrigo y Zacarias 2007, Zhou et al. 2010). En frutos de bananos, el efecto de aplicación de etileno a diferentes temperaturas evidencian que cercanas a $20^{\circ} \mathrm{C}$, durante 3 días ocasionan una adecuada degradación de clorofila y desarrollo de color amarillo. Caso contrario, sucedió cuando la aplicación de la hormona se realizó a $30^{\circ} \mathrm{C}$, ya que demostró que los procesos relacionados al cambio de color en estos frutos requieren de temperaturas cercanas a $\operatorname{los} 20^{\circ} \mathrm{C}$ (Du et al. 2014). Los cambios de color de la pulpa se deben, según Shah et al. (2015), a la síntesis de carotenos, y a procesos oxidativos mediados por enzimas, aunque también a la acumulación de líquidos en espacios intercelulares conforme la fruta envejece.

En piña, Liu y Liu (2014) reportaron que mantener frutos de piña, durante 36 horas a temperaturas entre 27 y $32^{\circ} \mathrm{C}$, ocasionaron un incremento en el valor de $\mathrm{a}^{*}$ (escala CIE $\mathrm{L}^{*} \mathrm{a}$ b) relacionado a una mayor degradación de clorofila y expresión de carotenoides lo que incrementó el color amarillo. Por otra parte, Solano-Maroto (2015) reportó un efecto positivo en el desarrollo de color amarillo en la epidermis de los frutos de piña, al mantenerlos durante 48 horas a temperatura ambiente, antes de ingresar al proceso poscosecha y posterior almacenamiento a $7,0^{\circ} \mathrm{C}$.

Debido a lo expuesto anteriormente, en relación con el efecto de la temperatura sobre la degradación de clorofila en la cáscara de frutos de piña y al hecho que este proceso presenta un rango de temperaturas óptimas para que suceda, se planteó evaluar el efecto de la variación en las temperaturas de acondicionamiento previas al almacenamiento a $7,5^{\circ} \mathrm{C}$, sobre la expresión de color amarillo de la calidad de los frutos de piña cv. Dorada Extra Dulce.

\section{MATERIALES Y MÉTODOS}

\section{Material vegetal y tratamientos}

Para la realización de este trabajo se utilizaron frutos de piña cv. Dorada Extra Dulce con calidad de exportación, procedentes de una finca comercial en Pital, San Carlos, Alajuela, Costa Rica. Las frutas se colocaron en diferentes temperaturas de acondicionamiento durante 48 horas, previo al almacenamiento a $7,5^{\circ} \mathrm{C}$ durante 21 días. Dicho trabajo se repitió 3 veces en el tiempo, durante julio y octubre del 2015, y marzo del 2016.

Los frutos seleccionados fueron producidos, cosechados y empacados según las prácticas comerciales vigentes para la exportación de piña. El proceso poscosecha incluyó la desinfección con cloro (120-150 mg. $\left.\mathrm{l}^{-1}\right)$, aplicación de cera a base de ácidos grasos y fungicida en el pedúnculo. Se seleccionaron frutas de calibre 7 con un grado de color de 0 , según la escala comercial, denominada Guía de Color Externo, la cual consiste de 6 categorías con un máximo de 3 grados (Figura 1) y tienen de un 95 a $100 \%$ de color verde en la epidermis. Se seleccionaron frutos en color 0 , por cuanto es el estado con el menor coeficiente de variación al ser seleccionado en empacadora. Esto permitió evitar el error experimental generado por los coeficientes de variación más altos obtenidos para colores externos de fruta superiores. 


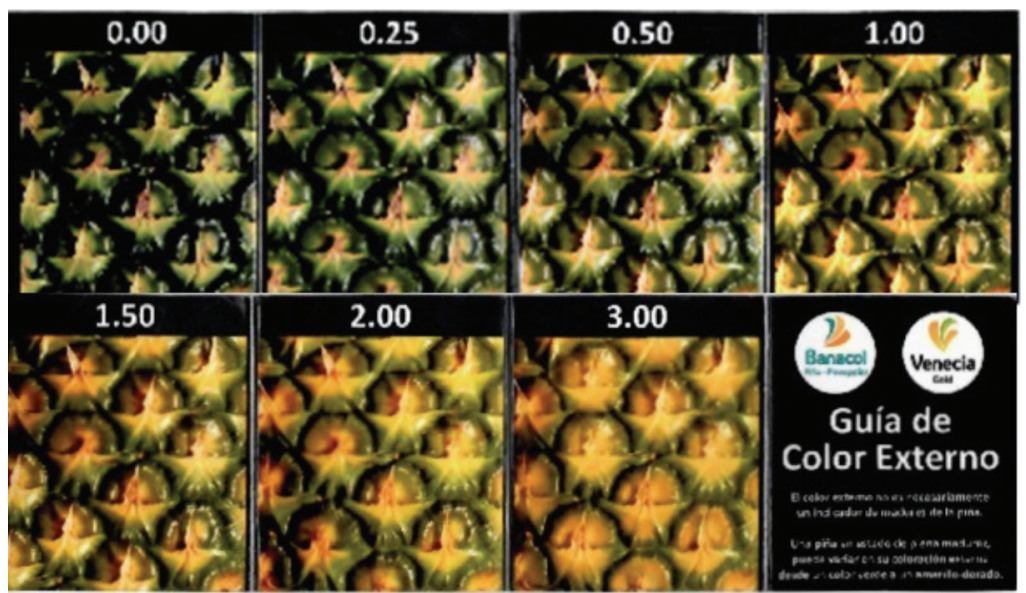

Figura 1. Escala de color externo de frutos de piña utilizada por la empresa proveedora de los frutos empleados para ensayo.

La fruta incluida en el ensayo se cosechó con un promedio de $12,1 \%$ de sólidos solubles totales y una translucidez de 0,5 , correspondiente a una fruta con pulpa que ya mostraba cambio de color hacia amarillo, con porosidad visible en general, opaca.

Los frutos se transportaron desde la planta empacadora hasta el Laboratorio de Tecnología Poscosecha del Centro de Investigaciones Agronómicas, de la Universidad de Costa Rica, Montes de Oca, lugar donde se establecieron los tratamientos. El tiempo de transporte fue de aproximadamente, 4 horas. Las temperaturas de acondicionamiento utilizadas durante las 48 horas correspondieron a $7,5^{\circ} \mathrm{C}, 10,0^{\circ} \mathrm{C}, 12,5^{\circ} \mathrm{C}$, $15,0^{\circ} \mathrm{C}, 17,5^{\circ} \mathrm{C}$ y $20,0^{\circ} \mathrm{C}$; posteriormente, todos los frutos se almacenaron a $7,5^{\circ} \mathrm{C}$ durante 21 días.

Se evaluó el color en la epidermis de la fruta, a través de la medición con colorímetro digital, la tasa respiratoria y la pérdida de peso; asimismo, se determinó la cobertura de moho en el pedúnculo. Al final del periodo de almacenamiento, se evaluó la firmeza. Solamente, en la segunda repetición, se evaluó el porcentaje de sólidos solubles y la acidez titulable. En la tercera repetición, se determinó la producción de etileno al final del periodo de variación en las temperaturas de acondicionamiento.
Se utilizaron 6 repeticiones por tratamiento, en donde cada repetición constó de una caja con 7 frutas (aproximadamente 10 kilogramos), que es la unidad comercial mínima. Para las evaluaciones de color en la epidermis, pérdida de peso y desarrollo de patógenos, se evaluaron 6 frutas de cada repetición de manera individual. Para las variables de calidad interna (firmeza, \% sólidos solubles y acidez titulable), se evaluaron 3 de las 6 frutas por repetición. Y para las de respiración y producción de etileno, se tomó una fruta de cada repetición, y se utilizaron únicamente para evaluar ambas variables.

Determinación del color de la epidermis. El color de la epidermis se evaluó con colorímetro digital (Modelo WR-10, I-Wave, Shangai, China) basado en la escala CIE L*a*b*. Esta variable se reportó como índice de color, según lo expuesto por Jiménez-Cuesta et al. (1981), IC= $\left(\left(1000 \mathrm{x} \mathrm{a}^{*}\right) /\left(\mathrm{L}^{*} \mathrm{x} \mathrm{b}^{*}\right)\right)$.

El color de cada fruta evaluada se estimó como un promedio de la medición de 3 frutículos centrales y se eliminó previamente la bráctea. Las evaluaciones se realizaron a las 0 y 48 horas en que se realizó la variación en las temperaturas de acondicionamiento, a los 21 días de almacenamiento a $7,5^{\circ} \mathrm{C}$. 
Tasa de respiración. La tasa respiratoria fue medida en frutos recién llegados a planta empacadora y después que los frutos habían estabilizado la temperatura para cada uno de los tratamientos (24 horas). Para ello los frutos fueron colocados en recipientes herméticos de $30 \mathrm{~L}$ de capacidad, los cuales fueron cerrados durante 7-8 horas dentro de la cámara fría correspondiente a cada tratamiento. Terminado este lapso, se determinó la cantidad de Dióxido de Carbono $\left(\mathrm{CO}_{2}\right)$ producido. Para ello, se utilizó un analizador de gases Bridge Analyzer, MAP Headspace $\mathrm{O}_{2} / \mathrm{CO}_{2}$ Analyzer, (Modelo 900141,m Bridge Analyzers, Ohio, Estados Unidos). Previo a colocar las frutas en los recipientes, se obtuvo el peso (g) de estas. Se colocó una piña por recipiente y 6 recipientes por cada temperatura de acondicionamiento. Con la información de $\mathrm{CO}_{2}$ producido, se calculó la tasa respiratoria en $\mathrm{mL} \cdot \mathrm{kg}^{-1} \cdot \mathrm{h}^{-1} \mathrm{CO}_{2}$ según lo descrito por Umaña et al. (2011).

Tasa de producción de etileno. De las mismas muestras utilizadas para determinar la tasa de respiración, se tomaron aproximadamente $5 \mathrm{~mL}$ de aire con una jeringa desechable y se procedió a evaluar la cantidad de etileno presente. Para medir la cantidad de etileno, se inyectaron $2 \mathrm{~mL}$ de muestra en un cromatógrafo de gases (Modelo 7820, Agilent, California, Estados Unidos) equipado con un detector de ionización de flama (FID) y una columna HP-PLOT/Q de 30 metros de largo por $0,53 \mathrm{~mm}$ de diámetro interno. El equipo fue previamente calibrado con gases de referencia Praxair ${ }^{\circledR}$ con concentraciones de 0,527 $\mu \mathrm{L} . \mathrm{L}^{-1}$ y $5,4 \mu \mathrm{L} . \mathrm{L}^{-1}$ de etileno (en nitrógeno). Para el cálculo de concentración de las muestras, se utilizó el área de los picos respectivos. Este parámetro se determinó al finalizar la variación en las temperaturas de acondicionamiento. Se trabajó con 6 repeticiones (frutas) por cada temperatura de acondicionamiento.

Pérdida de peso. La pérdida de peso fue determinada como la reducción, en el peso del producto cosechado, y fue expresado como el porcentaje de pérdida con respecto al valor inicial. Este parámetro se calculó durante el periodo en que la fruta permaneció en la variación en las temperaturas de acondicionamiento y también se determinó el porcentaje de pérdida de peso acumulada, que involucró los 21 días a $7,5^{\circ} \mathrm{C}$, más las 48 horas en las diferentes temperaturas de acondicionamiento.

Cobertura de mohos en el pedúnculo. Las frutas fueron examinadas para determinar la incidencia y la severidad del desarrollo de patógenos en el pedúnculo. La incidencia corresponde a la presencia de patógenos y severidad expresada como porcentaje de área del tejido afectado. Las evaluaciones de crecimiento de microorganismos en el pedúnculo de las piñas se efectuaron a las 0 y 48 horas en que se realizó la variación en las temperaturas de acondicionamiento, así como a los 21 días de almacenamiento a $7,5^{\circ} \mathrm{C}$.

Calidad interna de la fruta. Previamente se eliminó la epidermis de la cara externa de la fruta en la caja, mediante un corte longitudinal, liso y recto. Las evaluaciones de calidad interna se realizaron al finalizar los 21 días de almacenamiento a $7,5^{\circ} \mathrm{C}$.

La firmeza de la pulpa se evaluó en 2 puntos equidistantes de la zona ecuatorial. Se utilizó un penetrómetro QA supplies modelo FT011, con una punta convexa de 7,93 $\mathrm{mm}$. Este parámetro fue expresado en Newton $(\mathrm{N})$.

Para evaluar el porcentaje de sólidos solubles y acidez titulable, se extrajo una sección de pulpa, de aproximadamente $3 \mathrm{~cm}$ de ancho, mediante un corte longitudinal; posteriormente, se trituró y homogenizó manualmente; además se filtró a través de 4 capas de gaza. Con el jugo obtenido, se determinó el porcentaje de sólidos solubles a través de un refractómetro digital marca Atago modelo PAL-1; asimismo, se obtuvo la acidez a través de una titulación con una solución de $\mathrm{NaOH}$ 0,1 molar, mediante el indicador Fenolftaleína al $1 \%$. El porcentaje de acidez titulable se reportó como porcentaje de ácido cítrico. Por otra parte la translucidez se evaluó con la metodología descrita por Paull y Chen (2003). 
Análisis estadístico. Para cada repetición en el tiempo, se trabajó con un diseño irrestricto al azar, con 6 tratamientos. Las diferencias entre los tratamientos, para cada parámetro evaluado, se analizaron por medio de un análisis de varianza (ANOVA) con una comparación de promedios mediante la prueba de Tukey $(\mathrm{p}=0,05)$.

Se realizó un análisis combinado, en el cual se utilizó un modelo de bloques completos al azar con 3 repeticiones en el tiempo. Cada repetición estuvo conformada por 6 tratamientos y cada tratamiento estuvo compuesto por 6 réplicas. Cada bloque corresponde a una repetición en el tiempo. Se realizó una comparación de promedios a través de la prueba de modelos lineales mixtos (MLM), con un nivel de significancia $\mathrm{p}=0,05$.

Los análisis estadísticos se realizaron mediante el software estadístico R, versión 3.2.5 "Very, Very Secure Dishes". Para efectos de este trabajo, los datos presentados y discutidos corresponden a los del análisis combinado.

\section{RESULTADOS}

En la Tabla 1, se muestra el índice de color $\left((1000 * a) /\left(L^{*} b\right)\right.$ en la epidermis de los frutos de piña en los diferentes momentos de evaluación. Los valores obtenidos a las 48 horas, que corresponden al final del periodo de variación en las temperaturas de acondicionamiento, evidencia un incremento significativo en dicha variable para los frutos que permanecieron a temperaturas superiores a $15,0^{\circ} \mathrm{C}$. La temperatura de $17,5^{\circ} \mathrm{C}$, fue la que presentó el mayor valor, con diferencias significativas entre los tratamientos $(p=0,001821)$. Asimismo, los frutos que se mantuvieron en las temperaturas inferiores a $15,0^{\circ} \mathrm{C}$ no evidenciaron cambios en el índice de color con respecto al valor inicial. Luego de 21 días a $7,5^{\circ} \mathrm{C}$, se evidenció, que aquellas frutas que permanecieron en las temperaturas de acondicionamiento de $17,5^{\circ} \mathrm{C}$ y $20,0^{\circ} \mathrm{C}$, presentaron un efecto positivo en el índice de color en la epidermis; las cuales mostraron los mayores valores, en dicha variable con diferencias significativas entre tratamientos ( $\mathrm{p}=4,71 \mathrm{e}-09)$.

Tabla 1. Índice de color $\left(\left(1000^{*} \mathrm{a}\right) /\left(\mathrm{L}^{*} \mathrm{~b}\right)\right)$ en la epidermis de frutos de piña expuestos a diferentes temperaturas de acondicionamiento previo al almacenamiento a $7,5^{\circ} \mathrm{C}$ por 21 días.

\begin{tabular}{|c|c|c|c|c|}
\hline \multirow{2}{*}{ Temperatura $\left({ }^{\circ} \mathrm{C}\right)$} & \multicolumn{2}{|c|}{$\begin{array}{l}\text { Índice de color } \\
\left.\left(\left(1000^{*} \mathrm{a}\right) / \mathrm{L} * \mathrm{~b}^{*}\right)\right)\end{array}$} & \multicolumn{2}{|c|}{$\begin{array}{l}\text { Equivalencia escala } \\
\text { comercial }\end{array}$} \\
\hline & Inicial* & 48 horas & 21 días & 21 días \\
\hline 7,5 & $-3,13 \mathrm{a} \dagger$ & $-3,13 a b$ & $-2,45 \mathrm{a}$ & 0,25 \\
\hline 10,0 & $-3,13 \mathrm{a}$ & $-3,13 a b$ & $-2,30 \mathrm{a}$ & 0,25 \\
\hline 12,5 & $-3,27 \mathrm{a}$ & $-3,27 \mathrm{a}$ & $-2,41 \mathrm{a}$ & 0,25 \\
\hline 15,0 & $-3,18 \mathrm{a}$ & $-2,65 a b$ & $-1,86 a b$ & 0,50 \\
\hline 17,5 & $-3,15 \mathrm{a}$ & $-2,47 b$ & $-0,85 b c$ & 1,00 \\
\hline 20,0 & $-3,21 \mathrm{a}$ & $-2,64 a b$ & $-0,17 \mathrm{c}$ & 1,50 \\
\hline
\end{tabular}

* Inicial corresponde a los 0 días, 48 horas corresponde al final de periodo de variación en las temperaturas de acondicionamiento, 21 días corresponde al final del periodo de almacenamiento a 7,5 $5^{\circ} \mathrm{C}$

$\dagger \quad$ Letras distintas indican diferencias significativas según prueba de MLM $(\mathrm{p}=0,05)$.

En la Figura 2, se muestra la tasa respiratoria $\left(\mathrm{mL} \cdot \mathrm{kg}^{-1} \cdot \mathrm{h}^{-1} \mathrm{CO}_{2}\right)$ de los frutos de piña, luego de permanecer 48 horas expuestos a diferentes temperaturas de acondicionamiento. Se evidenció que, conforme se aumentó la temperatura de acondicionamiento, se incrementó 
exponencialmente dicha variable $\left(\mathrm{R}^{2}=0,9770\right)$. Los valores se encontraron entre 2,5 y 18,2 $\mathrm{mL} \cdot \mathrm{kg}^{-1} \cdot \mathrm{h}^{-1} \mathrm{CO}_{2}$ para las temperaturas de $7,5^{\circ} \mathrm{C}$ y $20,0^{\circ} \mathrm{C}$, respectivamente, con diferencias estadísticas entre los tratamientos $(\mathrm{p}<2,2 \mathrm{e}-16)$.

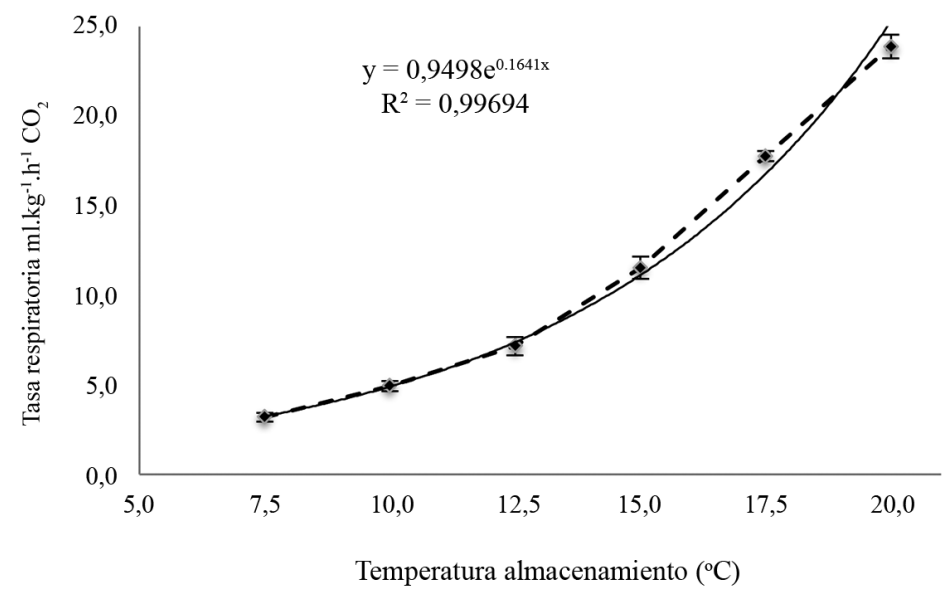

Figura 2. Tasa respiratoria $\left(\mathrm{mL} \cdot \mathrm{kg}^{-1} \cdot \mathrm{h}^{-1} \mathrm{CO}_{2}\right)$ de frutos de piña expuestos a diferentes temperaturas de acondicionamiento previo al almacenamiento a $7,5^{\circ} \mathrm{C}$ evaluada al finalizar 21 días de simulación de transporte. Barras verticales indican error estándar.

Después del periodo de exposición de 48 horas a diferentes temperaturas de acondicionamiento previo al almacenamiento a $7,5^{\circ} \mathrm{C}$ durante 21 días, no se logró detectar etileno en las muestras analizadas. Según los datos obtenidos, las diferentes temperaturas, a las cuales se expusieron los frutos durante 48 horas, no estimularon la producción endógena de etileno en valores superiores al límite mínimo de detección del equipo, el cual es de $0,25 \mu \mathrm{L} . \mathrm{L}^{-1}$ (datos no presentados).

En la Figura 2, se muestra el porcentaje de pérdida de peso en ambos periodos de evaluación. Durante las 48 horas, en que los frutos permanecieron en diferentes temperaturas de acondicionamiento. Se evidenció un aumento en la pérdida de peso, al incrementar las temperaturas a las cuales se expusieron las piñas (Figura 3A), con un incremento lineal del mismo $\left(\mathrm{R}^{2}=0,9049\right)$. En este caso, la pérdida de peso varió desde $0,5 \%$, en la temperatura de $7,5^{\circ} \mathrm{C}$, hasta $1,3 \%$ en la temperatura de $20,0^{\circ} \mathrm{C}$, con diferencias significativas entre las temperaturas $(\mathrm{p}=2,20 \mathrm{e}-16)$. El mismo comportamiento, descrito anteriormente, presentó la pérdida de peso acumulada, que incluye las 48 horas en temperaturas de acondicionamiento más 21 días a $7,5^{\circ} \mathrm{C}$ (Figura 3B). Esto muestra un incremento lineal en la variable en aquellos frutos que fueron sometidos a temperaturas mayores, previo al almacenamiento en frío, con un ajuste lineal $\left(\mathrm{R}^{2}=0,8373\right)$ y con diferencias significativas entre ellos $(p=2,55 \mathrm{e}-07)$. 

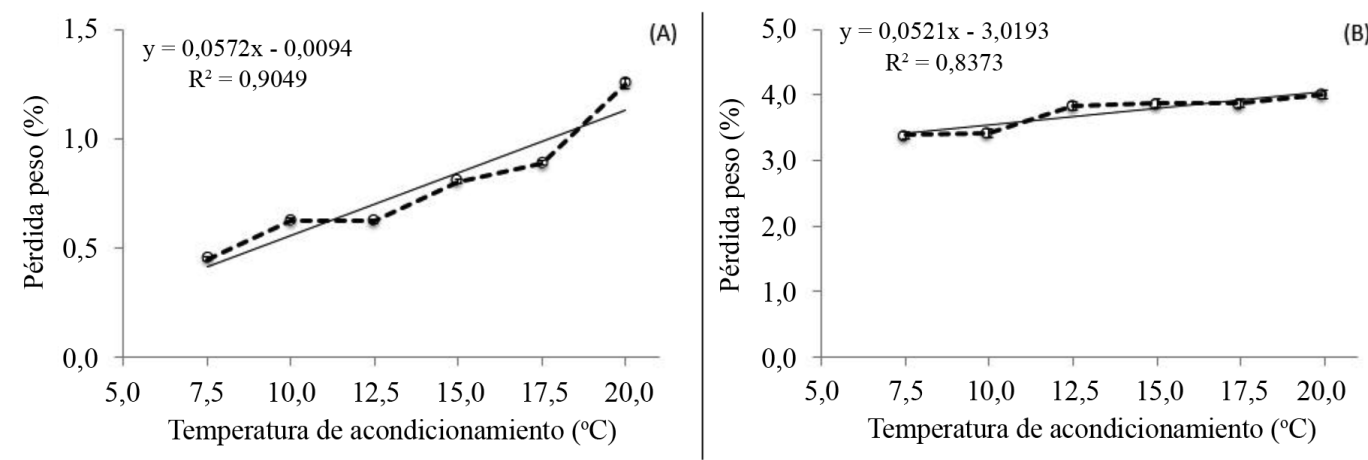

Figura 3. Pérdida de peso acumulado (\%) de frutos de piña. Al finalizar acondicionamiento por 48 horas a diferentes temperaturas (A). Al finalizar simulación de transporte a $7,5^{\circ} \mathrm{C}$ por 21 días (B). Barras verticales indican error estándar.

El porcentaje de incidencia de mohos y área del pedúnculo afectada por estos, se muestra en la Figura 4. Los datos corresponden a la evaluación, realizada al final de los 21 días a $7,5^{\circ} \mathrm{C}$; ya que en la evaluación realizada al finalizar la variación en las temperaturas de acondicionamiento durante 48 horas, el valor de incidencia y área afectada fue de $0 \%$. Se evidenció que ambas variables presentaron una correlación positiva con el aumento en las temperaturas a las que se expusieron los frutos previo al ingreso a $7,5^{\circ} \mathrm{C}$. Tal y como se muestra en la Figura 4A, el porcentaje de incidencia de mohos en el pedúnculo de las frutas, que se expusieron a temperaturas iguales o inferiores a $15,0^{\circ} \mathrm{C}$, durante 48 horas, previo al almacenamiento en frío, fue inferior a $20 \%$ y similares entre ellos. Asimismo, las piñas sometidas a temperaturas de $17,5^{\circ} \mathrm{C}$ y $20,0^{\circ} \mathrm{C}$, durante el mismo periodo, mostraron porcentajes de incidencia de 28 y $38 \%$, respectivamente, por lo que se presentaron diferencias significativas entre las temperaturas empleadas $(\mathrm{p}=8,21 \mathrm{e}-06)$. Con los resultados obtenidos, la temperatura más alta, que no induce aumento de incidencia o área afectada por mohos, fue la de $15^{\circ} \mathrm{C}$.
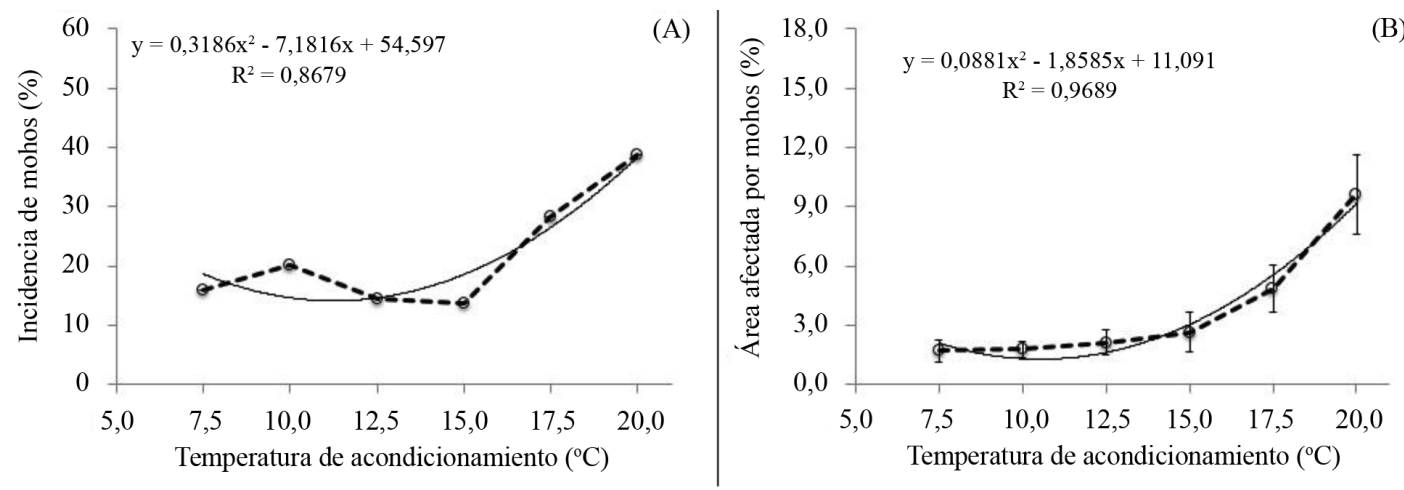

Figura 4. Desarrollo de mohos en el pedúnculo de frutos de piña expuestos a diferentes temperaturas de acondicionamiento previo al almacenamiento a $7,5^{\circ} \mathrm{C}$. Incidencia (A). Porcentaje de área afectada (B). Barras verticales indican error estándar. 
En cuanto al área del pedúnculo afectado por el desarrollo de mohos (Figura 4B), se evidenció que este valor incrementó al aumentar las temperaturas a las cuales se expusieron los frutos antes de permanecer 21 días a $7,5^{\circ} \mathrm{C}$. Aquellas piñas que permanecieron 48 horas a $20,0^{\circ} \mathrm{C}$ presentaron valores cercanos a $10 \%$; mientras que los demás frutos, que permanecieron en temperaturas iguales o inferiores a $17,5^{\circ} \mathrm{C}$, mostraron menos de un 5\% de afectación. Únicamente, el tratamiento de $20,0^{\circ} \mathrm{C}$ fue estadísticamente diferente a los demás tratamientos $(\mathrm{p}=0,001404)$.

En la Tabla 2, se muestran los datos de firmeza, sólidos solubles (\%) y acidez titulable (\%) de los frutos al finalizar los 21 días a $7,5^{\circ} \mathrm{C}$. En cuanto a la firmeza de la pulpa, esta varió desde $16,3 \mathrm{~N}$ hasta 17,5 $\mathrm{N}$ con diferencias significativas entre tratamientos $(\mathrm{p}=0,035)$, sin evidenciar un efecto significativo de las temperaturas de acondicionamiento sobre dicha variable. Respecto a los sólidos solubles, estos se encontraron entre 12,1 y $13,3 \%$ y sin diferencias estadísticas entre los tratamientos. La acidez titulable presentó un comportamiento similar al de la firmeza, en donde las temperaturas de acondicionamiento no tuvieron un efecto significativo sobre esta variable; sin embargo, se observaron diferencias estadísticas entre los tratamientos $(\mathrm{p}=0,0098)$, el menor valor fue $0,77 \%$ y el mayor $0,89 \%$.

Tabla 2. Firmeza, sólidos solubles (\%) y acidez titulable (\%) en la pulpa de frutos de piña expuestos a diferentes temperaturas de acondicionamiento previo al almacenamiento a $7,5^{\circ} \mathrm{C}$ durante 21 días.

\begin{tabular}{ccccc}
\hline \multirow{2}{*}{ Temperatura $\left({ }^{\circ} \mathrm{C}\right)$} & \multicolumn{4}{c}{ Calidad interna de la fruta } \\
\cline { 2 - 5 } & Firmeza (N) & Sólidos solubles (\%) & Acidez titulable (\%) & Translucidez \\
\hline 7,5 & $16,3 \mathrm{a}$ & $12,1 \mathrm{a}$ & $0,78 \mathrm{ab}$ & $0,75 \mathrm{a}$ \\
10,0 & $17,2 \mathrm{ab}$ & $12,4 \mathrm{a}$ & $0,77 \mathrm{a}$ & $0,75 \mathrm{a}$ \\
12,5 & $17,5 \mathrm{~b}$ & $13,2 \mathrm{a}$ & $0,85 \mathrm{ab}$ & $0,50 \mathrm{a}$ \\
15,0 & $16,5 \mathrm{ab}$ & $12,1 \mathrm{a}$ & $0,89 \mathrm{~b}$ & $0,75 \mathrm{a}$ \\
17,5 & $17,1 \mathrm{ab}$ & $13,3 \mathrm{a}$ & $0,78 \mathrm{ab}$ & $0,75 \mathrm{a}$ \\
20,0 & $16,9 \mathrm{ab}$ & $13,1 \mathrm{a}$ & $0,84 \mathrm{ab}$ & $0,50 \mathrm{a}$ \\
\hline
\end{tabular}

* Medias dentro de la misma columna con distinta letra difieren significativamente según prueba de MLM ( $\mathrm{p}=0,05)$ o Tukey $(\mathrm{p}=0,05)$.

\section{DISCUSIÓN}

El mantener frutos de piña durante 48 horas a temperaturas de $17,5^{\circ} \mathrm{C}$ y $20,0^{\circ} \mathrm{C}$ previo al almacenamiento durante 21 días a $7,5^{\circ} \mathrm{C}$, evidenció un efecto positivo en el cambio de color en la epidermis, ya que incrementó los valores de índice de color de manera significativa. Dichos frutos mostraron valores de $-0,85 \mathrm{y}-0,17$, respectivamente, al final del almacenamiento durante 21 días a 7,5 ${ }^{\circ} \mathrm{C}$ (Tabla 1). Valores de índice de color $\left((1000 * \mathrm{a}) /\left(\mathrm{L}^{*} \mathrm{~b}\right)\right)$, cercanos a 0,00 , corresponden a colores amarillos, lo que demuestra la pérdida de color verde en la epidermis de estos frutos (Jiménez-Cuesta et al. 1981).

Brat et al. (2004) y Hassan y Othman (2011) reconocen que el cambio de color en la epidermis de la piña se debe la degradación de clorofila y a la expresión de carotenoides y antocianinas en una relación adecuada, lo cual ha sido confirmado en el cv. Dorada Extra Dulce por Ulloa et al. (2015). La degradación de la clorofila es un proceso catabólico en el que intervienen diversas enzimas, lo cual da como resultado una disminución significativa en el contenido de dicho pigmento, permite el desenmascaramiento 
de los carotenoides y antocianinas, así como conlleva al cambio de color de verde a amarillo (Guevara y Jiménez 2006, Roca y Mínguez 2006, Cheng et al. 2012, Du et al. 2014, Kaewsuksaeng et al. 2015).

Los datos de este trabajo sugieren que el cambio de color en la epidermis de piña relacionado a la degradación de clorofila, puede inducirse durante las 48 horas de acondicionamiento en las temperaturas de $17,5^{\circ} \mathrm{C}$ y $20,0^{\circ} \mathrm{C}$, previas al almacenamiento en frío. Como ejemplo, en el caso de banano, la inducción y expresión genética relacionada a la degradación de clorofila, y cambio de color en la epidermis, requiere de temperaturas cercanas a los $20^{\circ} \mathrm{C}$ durante los primeros 3 días en que se aplica el tratamiento de maduración (Du et al. 2014). Matsumoto et al. (2009) reportaron que para el caso de mandarinas, la temperatura óptima para la degradación de clorofila y síntesis de carotenoides, relacionado al cambio de color de verde a anaranjado, es de $20^{\circ} \mathrm{C}$. Por su parte, los frutos de piña que se mantuvieron en temperaturas iguales o inferiores a $15,0^{\circ} \mathrm{C}$, previo al almacenamiento a $7,5^{\circ} \mathrm{C}$, no evidenciaron pérdida de color verde; posiblemente dichas temperaturas no son las adecuadas para permitir la inducción de cambio de color ligado a la degradación de clorofila.

La temperatura de $7,5^{\circ} \mathrm{C}$ durante 21 días limitó el cambio de color relacionado a la pérdida de clorofila, aunque no detuvo la expresión enzimática que condujo a dicho proceso en los frutos de $17,5^{\circ} \mathrm{C}$ y $20,0^{\circ} \mathrm{C}$. Lo anterior, puede relacionarse al efecto de la temperatura sobre la velocidad de reacción y la energía de activación que interactúan en las reacciones bioquímicas (Kubo 2015, Nelson y Cox 2015).

La tasa respiratoria de los frutos incrementó de manera exponencial al aumentar la temperatura a la que se expusieron durante 48 horas (Figura 1). Lo cual está relacionado al efecto de la temperatura sobre parámetros bioquímicos de las reacciones enzimáticas, principalmente la energía de activación. Así, al incrementar la temperatura a que se expone un fruto, se disminuye la energía de activación, lo cual aumenta las reacciones enzimáticas (Nelson y Cox 2015). En este caso, aumenta las reacciones metabólicas relacionadas a la respiración, las cuales dan la producción de energía (ATP) para mantener el fruto en adecuadas condiciones.

Los resultados obtenidos en este trabajo indican que las temperaturas a las cuales se expusieron los frutos de piña durante 48 horas, previas al almacenamiento durante 21 días a $7,5^{\circ} \mathrm{C}$, no estimularon la producción endógena de etileno en la fruta en concentraciones mayores al límite de detección del método y equipo empleado $\left(0,25 \mu \mathrm{L} \cdot \mathrm{L}^{-1}\right)$. Esto confirma que dicha fruta presenta una tasa baja de producción de la hormona (Kader 2002, Hassan y Othman 2011).

La pérdida de peso estuvo influenciada por la exposición a las temperaturas de acondicionamiento, durante 48 horas, previas al almacenamiento en frío durante 21 días (Figura 2), lo cual está relacionado a una mayor tasa de transpiración influenciada por las temperaturas utilizadas, ya que produjo mayor pérdida de agua con la consecuente pérdida de peso fisiológico (Kader 2002, Gopala-Rao 2015). Asimismo, los frutos que presentaron mayor tasa respiratoria durante la variación en las temperaturas de acondicionamiento, evidenciaron mayor pérdida de peso. Sin embargo, la pérdida de peso acumulada fue inferior a $5 \%$, el cual es el límite máximo permitido comercialmente.

Conforme se incrementó la temperatura a la que se expusieron los frutos durante 48 horas, se observaron incrementos en las variables de incidencia y área del pedúnculo afectada por mohos (Figura 3). La temperatura de $20,0^{\circ} \mathrm{C}$ fue la que presentó mayores valores para ambas variables. En frutos de piña, los principales patógenos relacionados a dicho problema son especies de los géneros Penicillium y Fusarium (Castro-Chinchilla y Umaña-Rojas 2015). Estos presentaron temperaturas óptimas de crecimiento cercanas a $20^{\circ} \mathrm{C}$; para el caso del primero, la temperatura óptima se encuentra entre 24 y $27^{\circ} \mathrm{C}$, y para el segundo el rango es de 20 a $25^{\circ} \mathrm{C}$ (Baert et al. 2007, Palmero et al. 2013). 
El crecimiento de patógenos, durante el almacenamiento en frío (21 días a $7,5^{\circ} \mathrm{C}$ ) se debe a que estos fueron capaces de continuar con su crecimiento aun en temperaturas inferiores a $5^{\circ} \mathrm{C}$; además, estas condiciones no ocasionan perdida en la viabilidad de dichos patógenos (Baert et al. 2008, Morales et al. 2008, Palmero et al. 2013). Asimismo, el pedúnculo de la fruta se convierte en un medio adecuado para el crecimiento de los microorganismos, debido a que es una fuente de exudados ricos en nutrientes (Castro-Chinchilla y Umaña-Rojas 2015). De acuerdo con los resultados obtenidos, la temperatura de $15^{\circ} \mathrm{C}$ no estimuló el desarrollo de mohos, sí permitió el desarrollo de color amarillo de la epidermis.

Los datos de este trabajo sugieren que las temperaturas a las cuales fueron expuestos los frutos durante 48 horas, previas al almacenamiento durante 21 días a $7,5^{\circ} \mathrm{C}$, no presentaron influencia en la calidad interna de las frutas (firmeza, sólidos solubles, acidez titulable, translucidez). No se observaron diferencias que se puedan atribuir a las condiciones experimentales para ninguna de las variables de calidad interna analizadas. Se evidencia que las temperaturas superiores a $12,5^{\circ} \mathrm{C}$ de acondicionamiento, previo al almacenamiento durante 21 días a $7,5^{\circ} \mathrm{C}$ ocasionan un efecto positivo en el aumento del índice de color. Sin embargo, temperaturas superiores a $15^{\circ} \mathrm{C}$ ocasionan incrementos en la tasa respiratoria y la pérdida de peso, además, de incrementar el desarrollo de mohos en el pedúnculo. No obstante, ninguna de las temperaturas empleadas ocasionan un efecto significativo en la calidad de las frutas. Según lo anterior, el uso de temperaturas cercanas a $15,0^{\circ} \mathrm{C}$ por 48 horas, previo al almacenamiento a $7,5^{\circ} \mathrm{C}$, podría ser de utilidad para mejorar el desarrollo de color en la epidermis de frutos cv. Dorada Extra Dulce, sin tener efecto negativo en la calidad final del producto, especialmente sin efecto sobre el desarrollo de mohos en el pedúnculo de cosecha. Ese aspecto podría permitir una única cosecha temprana de toda la fruta en un lote y desarrollar el color de la epidermis en la fase de almacenamiento.

\section{AGRADECIMIENTOS}

Al Sr. Francisco López Lee y a la empresa Inversiones y Procesadora Tropical (INPROTSA) por la ayuda brindada durante la ejecución de este trabajo.

\section{LITERATURA CITADA}

Baert, K; Devlieghere, F; Bo, L; Debevere, J; De Meulenaer, B. 2008. The effect of inoculum size on the growth of Penicillium expansum in apple. Food Microbiology 25:212-217.

Baert, K; Valero, A; De Meulenaer, B; Samapundo, S; Ahmed, M; Bo, L; Debevere, J; Devlieghere, F. 2007. Modeling the effect of temperature on growth rate and lag phase of Penicillium expansum in apple. International Journal of Food Microbiology 118:139-150.

Brat, P; Thi Hoang, L; Soler, A; Reynes, M; Brillouet, JM. 2004. Physicochemical characterization of a new pineapple hybrid (FLHORAN41 Cv.) Journal of Agricultural and Food Chemistry 52(20):6170-6177.

Castro-Chinchilla, J; Umaña-Rojas, G. 2015. Poblaciones e identificación de los hongos causantes de mohos poscosecha en el pedúnculo de la piña, en dos zonas de Costa Rica. Agronomía Costarricense 39(3):61-77.

Cheng, Y; Dong, Y; Yan, H; Ge, W; Shen, C; Guan, J; Liu, L; Zhang, Y. 2012. Effects of 1-MCP on chlorophyll degradation pathway-associated genes expression and chloroplast ultrastructure during the peel yellowing of Chinese pear fruits in storage. Food Chemistry 135:415-422.

Du, L; Yang, X; Song, J; Ma, Z; Zhang, Z; Pang, X. 2014. Characterization of the stage dependency of high temperature on green ripening reveals a distinct chlorophyll degradation regulation in banana fruit. Scientia Horticulturae 180:139-146.

Garita-Coto, Ra. 2014. La piña. Cartago, Costa Rica, Editorial Tecnológica de Costa Rica. 568 p.

Gopala-Rao, C. 2015. Engineering for storage of fruits and vegetables: cold storage, controlled atmosphere storage, modified atmosphere storage. India, BS Publications. 859 p.

Guevara, E; Jiménez, V. 2006. Fisiología de la producción de los cultivos tropicales: La reproducción de las plantas. San José, Costa Rica, Editorial Universidad de Costa Rica. 87 p.

Hassan, A; Othman, Z. 2011. Pineapple (Ananas comosus L. Merr.). In Yahia, EM (eds.). Postharvest Biology and Technology of Tropical and Subtropical fruits. Cambridge, Woodhead Publ. p. 194-217. 
Jiménez-Cuesta, M; Cuquerella, J; Martínez-Jávega, JM. 1981. Determination of a color index for citrus degreening. Proc. Int. Soc. Citriculture 2:750-753.

Kader, A. 2002. Postharvest technology of horticultural crops. University of California. Agricultural and natural resources. 3 ed. Oakland, California, USA. 535 p.

Kaewsuksaeng, S; Tatmala, N; Srilaong, V; Pongprasert, N. 2015. Postharvest heat treatment delays chlorophyll degradation and maintains quality in Thai lime (Citrus aurantifolia Swingle cv. Paan) fruit. Postharvest Biology and Technology 100:1-7.

Kubo, Y. 2015. Ethylene, oxygen, carbon dioxide and temperature in postharvest physiology. In Kanayama, Y; Kochetov, A (eds.). Abiotic stress biology in horticultural plants. Japan, Springer. p. 17-33.

Liu, C; Liu, Y. 2014. Effects of elevated temperature postharvest on color aspect, physiochemical characteristics, and aroma components of pineapple fruits. Journal of Food Science 79(12): 2409-2414.

Ma, G; Zhang, L; Kato, M; Yamawaki, K; Kiriiwa, Y; Yahata, M; Ikoma, Y; Matsumoto, H. 2015. Effect of the combination of ethylene and red led light irradiation on carotenoid accumulation and carotenogenic gene expression in the flavedo of citrus fruit. Postharvest Biology and Technology 99:99-104.

Matsumoto, H; Ikoma, Y; Kato, M; Nakajima, N; Hasegawa, Y. 2009. Effect of postharvest temperature and ethylene on carotenoid accumulation in the flavedo and juice sacs of Satsuma Mandarin (Citrus unshiu Marc.) fruit. Journal of Agricultural and Food Chemistry 57:4724-4732.

Morales, H; Sanchis, V; Coromines, J; Ramos, AJ; Marín, S. 2008. Inoculum size and intraspecific interactions affects Penicillium expansum growth and patulin accumulation in apples. Food Microbiology 25:378-385.

Nelson, D; Cox, M. 2015. Lehninger: Principios de Bioquímica. Cuchillo Foix, C; Suau León, P; Vendrell Roca, J Trad(s). 6 ed. Barcelona, España, Ediciones Omega. p.189-242.

Palmero-Llamas, D; Gálvez-Patón, L; García-Díaz, M; Gil-Serna, J; Sáez, B. 2013. The effects of storage duration, temperature and cultivar on the severity of garlic clove rot caused by Fusarium proliferatum. Postharvest Biology and Technology 78:34-39.

Paull, C; Chen, CC. 2003. Postharvest physiology, handling and storage of pineapple. In Bartholomew, DP; Paull, RE; Rohrbach, KG. (eds.). The pineapple: Botany, production and uses. Oxfordshire, Reino Unido, CABI Publising. p. 253-279.

Roca, M; Mínguez, MI. 2006. Chlorophyll catabolism pathway in fruits of Capsicum annuum (L): Staygreen versus red fruits. Journal of Agricultural and Food Chemistry 54(11):4035-4040.

Rodrigo, MJ; Zacarías, L. 2007. Effect of postharvest ethylene treatment on carotenoid accumulation and the expression of carotenoid biosynthetic genes in the flavedo of orange (Citrus sinensis L. Osbeck) fruit. Postharvest Biology and Technology 43:14-22.

Shah, YA; Ahiduzzaman, SA; Abdul, MB; Nafis, I; Jakaria; CO; Hafizur, R. 2015. Comparative effects on storage period of varieties pineapple fruits. Res. Agric. Livest. Fish. 2(3):395-410.

Solano-Maroto, J. 2015. Efecto del tiempo transcurrido entre cosecha e inicio de enfriamiento en túneles de aire forzado, sobre la vida útil de almacenamiento de piña (Ananas comosus) Var. Dorada Extra Dulce, en dos zonas de Costa Rica. Tesis Lic. San José, Costa Rica, Universidad de Costa Rica. 92 p.

Ulloa, L; Sáenz, MV; Castro, J. 2015. Efecto del almacenamiento a diferentes temperaturas sobre el desarrollo de color externo y la calidad de frutos de piña cv. Dorada Extra Dulce. Agronomía Costarricense 39(3):9-20.

Umaña, G; Loria, CL; Gómez, JC. 2011. Efecto del grado de madurez y las condiciones de almacenamiento sobre las características fisicoquímicas de la papaya híbrido Pococí. Agronomía Costarricense 35(2):61-73.

Zhou, JY; Sun, CD; Zhang, LL; Dai, X; Xu, CJ; Chen, KS. 2010. Preferential accumulation of orange-colored carotenoids in Ponkan (Citrus reticulata) fruit peel following postharvest application of ethylene or ethephon. Scientia Horticulturae 126:229-235. 